\title{
Intervenções Cognitivo-Comportamentais no Tratamento das Dependências Químicas
}

\author{
Marcelo Morandi ${ }^{1}$; Lívia Pires Guimarães ${ }^{2}$
}

\begin{abstract}
Resumo: O campo das dependências químicas vem recebendo importante atenção enquanto problema de saúde pública, ao passo que ocupa ponto de destaque nos debates sobre cuidados e intervenções possíveis. Neste contexto, o presente artigo tem como proposta confirmar o lugar privilegiado que as terapias comportamentais e cognitivas ocupam enquanto proposta de atenção a sujeitos usuários de drogas, apontando para técnicas de resultados cientificamente comprovados, e conclui que esta prática ainda detém lugar importante de destaque no campo de ferramentas de ajuda para esta clientela.
\end{abstract}

Palavras-chave: Dependência Química. Tratamento. Terapia Comportamental. Terapia Cognitiva

\section{Cognitive-Behavioral Interventions in the Treatment of Chemical Dependencies}

\begin{abstract}
The field of addictions has received significant attention as a public health problem, while occupying prominent point in discussions about care and possible interventions. In this context, this article aims to confirm the privileged place that behavioral and cognitive therapies occupy as a proposal for attention to subject drug users, pointing techniques scientifically proven results, and concludes that this practice still has an important place in the field of tools to help these clients.
\end{abstract}

Keywords: Chemical Dependence. Treatment. Behavioral Therapy. Cognitive Therapy.

\section{Introdução}

Segundo definição da Organização Mundial de Saúde (OMS), dependência química é um estado caracterizado pelo uso descontrolado de uma ou mais substâncias químicas psicoativas com repercussões negativas em uma ou mais áreas da vida do indivíduo, causando algum tipo de alteração em seu funcionamento. Ainda de acordo com a OMS, a dependência química consiste em uma doença crônica incurável, tornando-se um dos maiores desafios dos sistemas de saúde em todo o mundo, mas que pode ser tratada de forma efetiva. Este tratamento deve envolver, prioritariamente, evidências científicas e necessidades clínicas e técnicas empiricamente embasadas (DIEHL, 2011).

Dobson (2010) observou que demandas pela utilização de intervenções eficazes para problemas de saúde mental que incluam a busca de dados empíricos de resultados têm aumentado nos sistemas de saúde em todo o mundo.

\footnotetext{
${ }^{1}$ Psicólogo. Especialista em Dependência Química (PUC - MINAS); Psicoterapeuta Cognitivo-Comportamental (Amban-Ipq-USP) ; Psicólogo Clínico no Centro Mineiro de Toxicomania (Caps-ad, BH). E-mail: psicologomarcelomorandi@gmail.com;

${ }^{2}$ Psicóloga, Especialista em Dependência Química (UNIAD), em Criminologia (PUC-Minas/ACADEPOL), e em Gestão Pública em Organizações de Saúde (UFJF), Mestre em Educação, Cultura e Organizações Sociais (UFJF), Professora e Coordenadora do Curso de Especialização em Dependência Química (PUC-Minas). E-mail: liviapiresg@ yahoo.com.br.
} 
$\mathrm{O}$ autor ainda expõe demandas que apontam as terapias cognitivo-comportamentais (TCCs) como tratamento psicológico mais adequado para o transtorno por uso de substâncias. Em recente publicação, Cottraux (2007) apresenta estudos que também atestam a eficácia das TCCs, elevando-as ao status de mais importantes e mais bem validadas entre as abordagens psicoterápicas.

Estudos e achados de Robins et al. (1999) já haviam encontrado um crescimento na popularidade das TCCs na década de 80. Norcross et al. (2010) confirmaram a predominância das teorias cognitivocomportamentais como tratamento padrão para diversos transtornos.

As TCCs surgiram a partir de determinados fatores históricos contextuais, como da insatisfação com o behaviorismo clássico-metodológico baseado em paradigmas de aprendizagem do tipo estímulo-resposta (E-R), e com o modelo psicanalítico de terapia (DOBSON, 1988).

O behaviorismo metodológico baseava-se no realismo, visão segundo a qual toda experiência é causada por um mundo real, exterior e separado do mundo subjetivo e interno (BAUM, 2011). Os modelos de condicionamento que fundamentavam as práticas da terapia puramente comportamentalista não eram suficientemente abrangentes para favorecer uma compreensão mais ampla de problemas emocionais complexos e seu tratamento, apontando para uma necessidade de se explicar como os seres humanos percebiam os estímulos e que significado tinha tal percepção (DOZOIS, 2006). Já Mahoney (1974) propunha que processos cognitivos mediavam o comportamento e que poderiam ser inferidos e sustentados cientificamente.

Sobre o modelo psicanalítico de terapia - prática que tem por objetivo ajudar o paciente a desenvolver "insight" de materiais ditos inconscientes, fundamentados em desejos infantis não resolvidos que se manifestariam através de sintomas (KAPLAN e SADOCK, 1997) - a crise da teoria clínica psicanalítica ocorrida nos Estados Unidos, no final dos anos 50, decorreu da insatisfação de muitos analistas, entre eles Albert Ellis e Aaron Beck. Tal crise decorre de questionamentos à teoria das pulsões enquanto conjunto de conceitos considerados errôneos e derivados de uma neurobiologia obsoleta (SEMERANI, 2002).

Na Inglaterra, Eysenck (1952) já havia publicado um trabalho que avaliou a eficácia das terapias de orientação analítica, não encontrando provas conclusivas de que a psicoterapia psicanalítica fosse mais efetiva do que a remissão espontânea. Rachman e Wilson (2004) também constataram a ausência de indícios aceitáveis que sustentassem a visão da psicanálise como abordagem de tratamento eficaz. Ao contrário da terapia de orientação psicanalítica, a TCC não postula estruturas inconscientes ou defesas específicas que bloqueiam os pensamentos da consciência (CLARK et al., 1999). Aqui não existe a pretensão em dizer que uma prática é melhor do que outra, pois como bem aponta Simon (1990, p.95): 
Pretender que a psicanálise é um procedimento melhor, superior, porque alcança níveis mais profundos e amplos do psiquismo do que outras abordagens, é frivolidade e arrogância. Seria análogo a pretender que o trabalho do intelectual é superior ao do camponês. Se são fruto da escolha livre do homem têm o mesmo valor. São apenas estilos diferentes de vida...

No entanto, Kaplan e Sadock (1997) apontam para o fato de que o papel das psicoterapias de orientação psicanalítica no tratamento das dependências é discutível. Os autores ainda advertem quanto à necessidade de um período significativo de abstinência, com importante redução de fenômenos de craving, para que uma intervenção analítica seja instituída. Sigmund Freud, pai da psicanálise, em carta a Ferenczi $(1916)^{6}$, menciona que: "Os viciados não se prestam ao tratamento psicanalítico. Frente à menor frustração, retomam o uso da droga e abandonam o tratamento". A psicanálise ocupa-se do tema das toxicomanias há quase 120 anos, e não foi, até hoje, capaz de produzir evidências contrárias a este pensamento de Freud, motivo da literatura psicanalítica sobre o tema ainda ser frágil e pouco profícua (RAMOS, 2011).

Hoje as TCCs são um sistema de psicoterapia aplicável a uma ampla gama de transtornos psicológicos e psiquiátricos, destacando-se no cenário contemporâneo internacional devido a características como validação empírica, eficácia comprovada, orientada para a resolução de problemas de maneira colaborativa e também para o desenvolvimento de habilidades, refletindo uma mudança de paradigma no campo das psicoterapias (SERRA, 2013). Kouimtsidis et. al. (2007) sugerem que cada paciente é considerado único, com fatores de risco e habilidades de enfrentamento diferentes, indicando que ao longo do tempo, o tratamento poderia ser considerado mais uma jornada de descoberta pessoal, não apenas um processo de recuperação.

Zanelatto (2013) ressalta que as TCCs têm sido referenciadas como abordagens seguras e eficazes no tratamento do transtorno por uso de substâncias, e a aplicação desse conjunto de técnicas no contexto da terapia tem se mostrado eficaz tanto para o alcance como para a manutenção da abstinência. E tendo como proposta a contribuição para o contínuo enriquecimento desta prática, o presente trabalho traz uma revisão bibliográfica da literatura, que, ao se debruçar sobre essas abordagens terapêuticas, foca dois grandes grupos de correntes teóricas: o behaviorismo radical de Burrhus Frederic Skinner e o modelo cognitivo de Aaron Temkin Beck, apresentando ainda os principais conceitos e técnicas criados com o objetivo de compreender e tratar os pacientes usuários de álcool e outras drogas.

Ainda como importante proposta, com este trabalho espera-se contribuir não somente para a prática das TCCs aplicadas ao tratamento das dependências químicas, mas também para a promoção de resultados duradouros relacionados à redução ou interrupção do consumo de drogas, melhora na

${ }^{6}$ JONES E. Vida e obra de Sigmund Freud. Buenos Aires: Horme, 1976. p. 204 
qualidade de vida e habilidades para lidar com as vicissitudes ocasionadas pelo comportamento aditivo.

\section{Intervenções Cognitivo-Comportamentais no Tratamento das Dependências Químicas}

Banaco (2013) explica que os modelos experimentais estão entre as principais estratégias utilizadas pelos analistas do comportamento para compreender melhor os transtornos psiquiátricos. Nesses modelos, buscam-se relações observadas entre as ações do organismo e mudanças no ambiente onde esse organismo vive. O autor ainda expõe que um dos tipos de interação estudados pelos modelos experimentais é aquele relacionado às mudanças no ambiente, chamados de estímulos. $\mathrm{O}$ organismo apresenta rápida adaptação a tais mudanças, o que é chamado de respostas. Esse fenômeno é abordado pelo estudo de um processo comportamental denominado pela literatura como respondente ou pavloviano (SIEGEL, 1979). Segundo Hartnoll (1991) não era a dependência física que explicaria, em primeira instância, o constante uso da droga, mas o esquema social de reforço por estímulos associados a ela.

Há outro tipo de interação relacionado a mudanças no ambiente causadas pela ação do organismo e que podem influenciar na probabilidade futura dessa ação. Nessa interação, as consequências da ação do organismo podem torná-la mais provável (i.e., ocorreria o fortalecimento da classe de respostas) ou menos provável (ocorreria, então, o enfraquecimento). À essa interação dá-se o nome de contingência operante (BANACO, 2013). Uma breve passagem retrata bem de que forma a corrente do behaviorismo radical analisa estas conexões dentro do contexto clínico:

\footnotetext{
Analistas do comportamento consideram dois aspectos importantes para a detecção e intervenção de problemas psiquiátricos: se o comportamento "transtornado" é uma resposta anormal do organismo a mudanças normais do ambiente ou se o comportamento problemático é uma resposta normal do organismo a uma situação ambiental que mudou de maneira exacerbada... (KUPFER e FALK, 1998, p. 334-51).
}

A teoria analítico-comportamental tem buscado muitas formas de interpretar o fenômeno da dependência química, sobretudo por entender que os comportamentos são multifuncionais, ou seja, tem muitas razões para acontecer (BANACO, 2013). Por esta razão, considera-se que o comportamento é determinado pela interação entre a ação do organismo e pela mudança ambiental produzida por esta ação; ações estas que podem ser efetivas em um ambiente e talvez não o sejam em outro, tornando o comportamento muito plástico, e sua análise bastante complexa. Esse tipo de comportamento pode ser observado apenas sob algumas circunstâncias. A condição para esclarecer as circunstâncias envolvidas no aparecimento do comportamento-problema é denominada análise 
funcional, que nada mais é do que o análogo experimental das condições envolvidas no modelo experimental (ANDERY et. al., 2001), ou seja, para entender o comportamento, temos que entender não só suas consequências, mas também o contexto em que ocorrem (BAUM, 2011).

A terapia comportamental (BT) é focada em estratégias para modificar e melhorar o estado motivacional (MILLER e ROLLNICK, 2001), explorando comportamentos associados ao uso de drogas, visando a mudança no estilo de vida e nos comportamentos de risco ao uso de substâncias (MARLATT e GORDON, 1994). Neste caso, junto com o paciente, o terapeuta comportamental faz um mapeamento sobre situações, lugares, companhias, etc, que estariam condicionados ao uso de drogas, ajudando o paciente a reconhecer estes determinantes e a traçar novos comportamentos, desfazendo estímulos que se condicionaram ao uso de substâncias (CHILDRESS et. al., 1993).

Já no campo da teoria cognitivista, Aaron Beck, considerado o pai da terapia cognitiva, também trouxe sua contribuição para o entendimento das dependências químicas. Para Silva (2013), o modelo cognitivo do uso de substâncias, ou modelo cognitivo de recaída, proposto por Beck em 1993, representa o modelo que melhor explica a gênese da drogadição. Segundo o autor, se existe fundamentação científica para mais de um modelo, talvez os melhores fossem aqueles que contemplassem aspectos como explicação satisfatória; maior intersecção entre os fenômenos que envolvem o consumo de drogas; menor número de contradições entre tais fenômenos; explicação tanto do desenvolvimento quanto da manutenção da dependência; suporte teórico às intervenções e técnicas nele apoiadas; e maior número de evidências científicas que os comprovem. Dentre os modelos psicológicos, o modelo de Aaron Beck preencheria os requisitos supracitados.

O modelo cognitivo de uso de substâncias de Beck et. al. (1993) diz de situações que agem como estímulo de alto risco (estímulos internos ou externos). Tais estímulos estariam diretamente ligados à ativação de crenças nucleares que o indivíduo carrega sobre si, o mundo e o futuro, além de pressupostos sobre o uso de drogas. Com estas crenças ativadas, eclodiriam pensamentos automáticos, que Beck também chama de pensamentos facilitadores ao uso de drogas, que eliciariam sinais e sintomas de fissura. Regido pelo craving e autorizado pelas crenças facilitadoras, o indivíduo planeja e providencia o acesso à droga ou ao comportamento e inicia seu uso. O uso da substância (ou o engajamento no comportamento aditivo) desencadearia uma situação contraditória: desejo de continuar o uso, por um lado, e sentimentos de culpa e fracasso, por outro. O desconforto psicológico ativaria mais crenças disfuncionais, seguidas de pensamentos permissivos, e o uso da droga ou o engajamento no comportamento teria continuidade.

Tratamentos de base operante, como a terapia comportamental de casal, o tratamento por reforço comunitário e o tratamento por manejo de contingências, alcançam maior destaque no tratamento das dependências químicas (MIGUEL e GAYA, 2013). Estes três métodos de tratamento acreditam que os comportamentos ligados ao consumo de substâncias sejam operantes, ou seja, 
comportamentos controlados pelas consequências que produzem. Por isso o foco das intervenções é modificar o ambiente do paciente de tal modo que ele seja mais reforçado por manter a abstinência do que por fazer uso de substâncias (MIGUEL e GAYA, 2013).

Baseada nos princípios comportamentais, a terapia comportamental de casal, sustenta que interações familiares disfuncionais produzem contingências que reforçam o consumo de álcool e outras substâncias. Desta maneira, o objetivo central dessa terapia seria modificar as contingências presentes na relação familiar, eliminando aquelas que reforçam o comportamento aditivo e estimulando novas contingências que reforçam comportamentos favoráveis à abstinência (FALSSTEWART e FARRELL, 2003).

Para Gaya (2013), as sessões devem ser altamente estruturadas, envolvendo os sentimentos, as habilidades de comunicação e atividades planejadas em comum. Estudos recentes de Fals-Stewart (2003) apontam que a terapia de casal produz melhores resultados do que tratamentos individuais tradicionais na promoção da abstinência (resultados obtidos durante o tratamento e até 12 meses após seu término). Importante ainda ressaltar que a abordagem é contraindicada em casos de relacionamentos destrutivos, nos quais são frequentes situações de violência física, e/ou quando um dos cônjuges apresenta medo constante do outro. É também contraindicada nos casos em que ambos os cônjuges são dependentes químicos (FALS-STEWART et. al., 1999).

Existe, atualmente, um número significativo de estudos que demonstram a importância do condicionamento operante para o entendimento da dependência de substâncias (HIGGINS e PETRY, 1999). Isso significa que contingências presentes no contexto socioambiental em que o indivíduo vive, atua de maneira direta no surgimento e na manutenção de comportamentos ligados ao consumo de drogas. Assim, para reduzir ou eliminar o uso de substâncias, é importante modificar as contingências presentes no ambiente social do indivíduo, a fim de que o comportamento de manutenção da abstinência seja mais reforçado que o comportamento de consumo (VUCHINICH e TUCKER, 1988).

Do ponto de vista teórico-metodológico da perspectiva comportamental, há duas formas em que se pode atuar sobre as contingências para eliminar o consumo de substâncias. Uma forma é aumentar as consequências aversivas relacionadas ao consumo de substâncias com o intuito de deixálas menos atrativas. Alternativamente a essa metodologia, existe o princípio que fundamenta o tratamento por reforço comunitário (TRC): em vez de punir o comportamento de usar a substância, são reforçados positivamente comportamentos alternativos ao uso (MIGUEL e GAYA, 2013).

O TRC pode ser caracterizado como um aconselhamento interventivo comportamental ou cognitivo-comportamental centrado em modificar o estilo de vida do usuário de substâncias (SMITH et. al., 2001). O TRC, portanto, transcende o setting terapêutico, tendo um olhar mais social cujo objetivo seria manipular algumas contingências para aumentar os reforçadores presentes nos ambientes profissional, familiar e recreativo, criando, como consequência, um novo estilo de vida 
mais reforçador do que aquele em que há o consumo de substâncias (SCHOTTENFELD, 2000). O TRC é composto de uma variedade de estratégias. O primeiro passo é aumentar a motivação do indivíduo em cessar o consumo de substâncias. Em geral, o terapeuta busca criar um vínculo positivo e de confiança com o paciente. Quanto melhor for este vínculo, maior será o impacto da fala do profissional. Nessa fase, podem ser usadas estratégias presentes no modelo da entrevista motivacional (MIGUEL e GAYA, 2013).

Em um estudo de metanálise organizado por Roozen et. al. (2004), em que foram incluídos apenas estudos randomizados controlados, demonstrou-se que existe forte evidência de que, para o tratamento do alcoolismo, o TRC é mais eficaz que o tratamento tradicional no que diz respeito aos desfechos: número de dias de abstinência, redução do consumo pesado e melhora na qualidade de vida. O mesmo estudo também traz indícios de que este modelo de terapia em associação com o manejo de contingências (MC) aparece como uma das intervenções mais eficazes na promoção da abstinência continuada de cocaína. Há ainda evidências empíricas de que tratamentos que manipulam o ambiente do usuário, criando novos reforçadores contingentes a comportamentos alternativos ao consumo de substâncias (como a terapia comportamental de casal e o TRC), são eficazes em reduzir o consumo, engajar o paciente no tratamento e promover melhoras significativas em diversas áreas de sua vida (família, trabalho, lazer, etc.) (MIGUEL e GAYA, 2013).

$\mathrm{O}$ tratamento por $\mathrm{MC}$ é, seguramente, o método de tratamento mais recente na literatura científica e na prática de serviços especializados, sendo, hoje, o tratamento de referência nos Estados Unidos e em alguns países europeus. Esse reconhecimento foi confirmado em estudos desenvolvidos por Higgins et. al. (1991). Tais estudos mostram que pacientes usuários de substâncias conseguem diminuir seu consumo e, às vezes, pará-lo quando algumas contingências de seu ambiente são manipuladas, aumentando a disponibilidade de reforçadores alternativos àqueles ligados ao uso de substâncias. Ainda segundo Higgens, o objetivo do tratamento seria promover a mudança de comportamentos relacionados ao uso de substâncias ao manipular certas contingências presentes no dia a dia do paciente.

Miguel e Gaya (2013) apontam para uma semelhança entre o TRC e o MC. Ambas as técnicas parecem idênticas, mas há uma diferença importante: enquanto o TRC procura reforçar comportamentos alternativos ao consumo de substâncias (p. ex., jogar bola com amigos, sair com os filhos), o tratamento por MC tenta reforçar comportamentos alternativos incompatíveis com aqueles ligados ao consumo de substâncias (p. ex., o comportamento de ficar abstinente é incompatível com o de consumo).

Estudos de revisão de literatura e metanálise apontam que o tratamento por MC, aplicado sozinho ou em conjunto com técnicas comportamentais e cognitivas, é a forma de tratamento mais eficaz em promover a abstinência continuada e garantir o paciente no tratamento (LUSSIER et. al., 
2006). O MC também foi estudado para o tratamento de dependências a diversas substâncias, como a cocaína, heroína, álcool, anfetamina, metanfetamina, maconha e tabaco, demonstrando eficácia na promoção de abstinência em todas as substâncias estudadas (DUTRA et. al., 2008).

Considerando o modelo cognitivo do abuso de substâncias, descrito por Beck et. al. (1993), as técnicas e estratégias cognitivas que serão descritas são consideradas as mais relevantes e as que demonstram maior eficácia para as necessidades dos pacientes dependentes (MORAES e BOSCHETTI, 2013). Ellis (1979) diferenciou as estratégias em dimensões "não refinadas" (centramse na mudança do conteúdo do pensamento mediante intervenções autoinstrutivas) e "refinadas" (introduzem processos de raciocínio mais sofisticados para mudar o conteúdo, o processo e a estrutura do pensamento mediante uma análise racional profunda).

As estratégias não refinadas são, de preferência, utilizadas na fase inicial de intervenção, pois funcionam melhor com indivíduos muito angustiados e com aqueles que estão em crise imediata (MORAES e BOSCHETTI, 2013). Dentre elas, a psicoeducação alcança maior destaque. O objetivo da intervenção psicoeducativa é familiarizar o paciente quanto ao Modelo Cognitivo e quanto ao funcionamento do transtorno aditivo. Fornece conhecimentos teóricos e práticos para que o paciente entenda as características do seu problema e as propostas psicoterápicas (KNAPP e BECK, 2008).

Dados epidemiológicos, consequências clínicas, neurobiologia da drogadição, prejuízos psicossociais devem ser apresentados de forma objetiva, oferecendo um panorama das complicações agudas e crônicas decorrentes do uso de drogas. Munido destas informações, o paciente terá a oportunidade de fazer escolhas mais precisas e realistas (RANGÉ et. al., 2011). Tal intervenção requer conhecimento e especialização por parte do profissional, devendo ser implantada logo no início do tratamento (ZANELATTO, 2013) e pode contribuir para evitar desejo subjetivo intenso no início da proposta de redução ou interrupção do consumo (MARINHO et. al., 2012). A ausência de um trabalho nesta área torna mais provável a ocorrência de lapsos ou recaídas devido à falta de compreensão quanto aos fatores envolvidos na perpetuação do uso (CARROLL, 1998).

As estratégias consideradas refinadas correspondem a uma reestruturação cognitiva mais profunda e devem ser evitadas: no início do tratamento, em situações de crise, em estágio motivacional de pré-contemplação ou quando o paciente apresenta déficit cognitivo importante (BECK, 2013). Por reestruturação cognitiva entende-se um processo onde as intervenções são feitas a nível mais estrutural, impactando na modificação de esquemas e crenças do paciente, garantindo benefícios a longo prazo. Esquemas são estruturas cognitivas que atribuem significado aos estímulos sensoriais (internos e externos) de forma automática e inconsciente. Crenças são proposições individuais, carregadas de valor de verdade para o próprio sujeito. Essas proposições são relativamente rígidas, supergeneralizadas, construídas desde a primeira infância, e refletem o modo como o sujeito enxerga a si mesmo, as pessoas, o mundo e o futuro. Levam ao sofrimento psíquico e comportamentos 
mal adaptados, além de impedirem a realização de metas. O esquema é a estrutura, e a crença seria o conteúdo semântico dessa estrutura, ou seja, o esquema colocado em palavras (BECK, 2013).

As estratégias refinadas que ganham maior destaque, relevância e que demonstram maior eficácia no tratamento das dependências químicas são o diálogo socrático, ou questionamento socrático como é mais conhecido, a reatribuição (torta de responsabilidade) e a flecha descendente (BOSCHETTI e MORAES, 2013).

O método socrático era praticado por Sócrates (469-399 a.C.), professor e filósofo grego, que colocava em dúvida crenças arraigadas dos seus contemporâneos. Sócrates acreditava que a prática disciplinada do questionamento consciente permitia ao indivíduo estudar ideias de maneira lógica para determinar sua validade. Para isso, ele usava perguntas simples e quase ingênuas, com o objetivo de revelar as contradições da atual forma de pensar de seus debatedores, normalmente baseada em valores e preconceitos da sociedade, redefinindo tais valores. O questionamento socrático foi incorporado à terapia cognitiva como estratégia central e durante o processo o terapeuta educa o paciente a considerar seus pensamentos que emergem automaticamente apenas como hipóteses, para, então, avaliá-los de maneira crítica e gerar pensamentos alternativos mais adaptativos (BOSCHETTI e MORAES, 2013).

Nesta proposta, o questionamento socrático aplicado ao tratamento das dependências químicas tem como principal objetivo desafiar mitos relacionados às expectativas de resultado positivo e discutir os componentes psicológicos e sociais do uso de substâncias, proporcionando ao paciente oportunidades de fazer escolhas com mais informações em situações de alto risco (MARLATT e DONOVAN, 2009), além de perceber e responder ao real de forma mais funcional e adaptativa (BECK, 2013).

A torta de responsabilidade (LEAHY, 2006) é considerada uma técnica de reatribuição de significado que auxilia o paciente a buscar explicações alternativas relacionadas à sua responsabilidade ou culpa pela ocorrência de um evento que tenha modulado negativamente seu humor, levando ao comportamento do uso de drogas. No exercício da reatribuição o paciente irá construir, colaborativamente com o terapeuta, explicações mais elaboradas sobre determinada experiência que gerou desconforto interno, listando possíveis causas que justifiquem a ocorrência de determinado evento. Em seguida, ainda com a ajuda do terapeuta, o paciente irá "fatiar a torta" utilizando-se da listagem das causas das sensações desconfortáveis, determinando a porcetagem de cada razão. O objetivo maior é ajudar o paciente a entender que cada evento é justificado pelo acúmulo de vários fatores que contribuem dentro de um todo, não sendo ele $100 \%$ responsável pelo que aconteceu.

A flecha ou seta descendente possibilita a identificação de esquemas disfuncionais a partir de um pensamento e ajuda na descoberta de medos subjacentes de um paciente (LEAHY, 2006). Quando 
ajudamos as pessoas a modificarem esquemas, estamos trabalhando nos alicerces de seu autoconceito e modo de viver no mundo. Esquemas são as crenças nucleares que contem as regras fundamentais para o processamento de informações (WRIGTH et. al., 2008).

De acordo com Beck (1993), o uso de substâncias é uma estratégia compensatória que procura anular crenças nucleares e as emoções ruins que as acompanham. As intervenções cognitivas objetivam modificar e reestruturar cada um desses grupos de crenças nucleares disfuncionais, diminuindo o craving e interrompendo o uso ou prevenindo a recaída (SILVA, 2013). Sendo assim, o trabalho de revisar esquemas disfuncionais pode gerar benefícios positivos em duas áreas principais: alívio dos sintomas atuais e melhor resistência a estressores no futuro (WRIGTH et. al., 2008)

A eficácia das intervenções cognitivas para o tratamento do abuso de substâncias foi amplamente demonstrada em diversos estudos, entre eles os de Beck et. al. (1993), Cordioli e Knapp (2008) e Carroll (1998), e os principais ganhos terapêuticos foram elevada taxa de adesão ao modelo de tratamento e manutenção da abstinência por um período maior de tempo.

\section{Considerações Finais}

Não há como pensar em um trabalho único para uma doença complexa, motivo pelo qual a prática das TCCs também podem estar associadas a outros modelos de intervenção como a terapia de família e/ou de casal, as terapias de grupo, a entrevista motivacional, a administração farmacológica, entre outras.

O que observamos nas literaturas atuais, é que a prática clínica das TCCs está em constante processo de reformulação e refinamento de seus conceitos teóricos e práticas de intervenção. Sendo assim, o presente trabalho não teve a intenção de esgotar o assunto ou elevar a prática das TCCs a um patamar estanque para o tratamento de comportamentos aditivos, mas confirmar a importância desta prática para este público que busca os serviços especializados na intenção de ter os seus padrões de comportamento e estilo de vida modificados, e para melhor. Pautando-se nesta lógica, e como já confirmado pela extensa literatura vigente sobre o assunto, as TCCs ainda ocupam um lugar importante enquanto ferramenta de ajuda para esta clientela.

\section{Referências}

ABREU, C. N.; GUILHARDI, H. J. Terapia comportamental e cognitivo-comportamental. São Paulo: Roca, 2004. 
ANDERY, M. A. P. et al. Análise funcional na análise do comportamento. In: GUILHARDI, H. J. et al. Sobre comportamento e cognição: expondo a variabilidade. Santo André: Esetec, 2001. p. 14857.

BANACO, R. A. Teoria comportamental. In: ZANELATTO, N. A.; LARANJEIRA, R. (Org.). O tratamento da dependência química e as terapias cognitivo-comportamentais: um guia para terapeutas. Porto Alegre: Artes Médicas, 2013. p. 135-151.

BAUM, W. M. Compreender o behaviorismo: comportamento, cultura e evolução. Porto Alegre: Artmed, 2008.

BECK, A. T. et al. Cognitive therapy of substance abuse. New York - London: Guilford Press, 1993.

BECK, J. S. Terapia cognitivo-comportamental: teoria e prática. 2. ed. Porto Alegre: Artes Médicas, 2013.

CARROLL, K. M.; NATIONAL INSTITUTE DRUG ABUSE. A cognitive behavioral approach: treating cocaine addiction. Rockville: NIDA, 1998.

CHILDRESS, A. R. et al. Cue reactivity and cue reactivity interventions in drug dependence. In: ONKEY L. S. et al. Behavioral Treatment for Drug Abuse and Dependence. Rockville, MD: National Institute on Drug Abuse; 1993:73-95. NIDA research Monograph Serie no 137.

CLARK, D. A. et al. Scientific foundations of cognitive theory and therapy of depression. New York: Wiley, 1999.

CORDIOLI, A. V.; KNAPP, P. A. A terapia cognitivo-comportamental no tratamento dos transtornos mentais. Revista Brasileira de Psiquiatria. v. 30, n. 2, p. 51-3, 2008.

COTTRAUX, J.; MATOS, M. G. Modelo europeu de formação e supervisão em terapias cognitivocomportamentais (TCCs) para profissionais de saúde mental. Revista Brasileira de Terapias Cognitivas. v. 3, n. 1, p. 49-61, 2007.

DIEHL, A. et al. Dependência química: prevenção, tratamento e políticas públicas. Porto Alegre: Artmed, 2011.

DOBSON, K. S.; BLOCK, L. Historical and philosophical bases of the cognitive-behavioural therapies. In: DOBSON, K. S., editor. Handbook of cognitive-behavioural therapies. New York: Guilford, 1988. p. 3-38.

DOBSON, D.; DOBSON, K. S. A terapia cognitivo-comportamental baseada em evidências. Porto Alegre: Artmed, 2010.

DOBSON, K. S.; SCHERRER, M. C. História e futuro das terapias cognitivo-comportamentais. In: KNAPP, P., editor. Terapia cognitivo-comportamental na prática psiquiátrica. Porto Alegre: Artmed, 2004. p. 42-57.

DOZOIS, K. S. Manual de terapias cognitivo-comportamentais. Porto Alegre: Artmed, 2006.

DUTRA, L. et al. A meta-analytic review of psychosocial interventions for substance use disorders. American Journal of Psychiatry, v. 165, n. 2, p. 179-187, 2008. 
ELLIS, A. Rational-emotive therapy as a new theory of personality and therapy. In: ELLIS, A.; WHITELEY, J. M., editors. Theoretical and empirical foundations rational-emotive therapy. New York: Brooks/Cole, 1979. p. 1-6.

FALS-STEWART, W.; O’FARRELL, T. J. Behavioral family counseling and naltrexone compliance for male opioid-dependent patients. Journal of Consulting and Clinical Psychology, v. 71, n. 3, p. $432,2003$.

FALS-STEWART, W. et al. Drug-abusing patients and their intimate partners: dyadic adjustment, relationship stability, and substance abuse. J Abnorm Psychol, 1999.

FALK, J. L.; KUPFER, A. S. Adjunctive behavior: application to the analysis and treatment of behavior problems. In: O`DONOHUE, W., editor. Learning and behavior therapy. Boston: Allyn \& Bacon, 1998. p.334-51.

FOCCHI, G. R. A. et al. Dependência química: novos modelos de tratamento. São Paulo: Roca, 2001.

HARTNOLL, R. The relevance of behavioural models of drug abuse and dependence liabilities to the understanding of drug misuse in humans. In: WILLNER P., editor. Behavioural models in psychopharmacology: theoretical, industrial and clinical perspectives. Cambridge: Cambridgy University, 1991. p. 503-19.

HIGGINS, S. T. et al. A behavioral approach to achieving initial cocaine abstinence. Am J Psychiatry, v. 148, n. 9, p. 1218-1224, 1991.

HIGGINS, S. T.; PETRY, M. N. Contingency management: incentives for sobriety. Alcohol Res Health, v. 23, n. 2, p. 122-7, 1999.

JERONIMO, C. S.; SERRA, A. M. Terapias cognitiva e cognitivo-comportamental em dependência química. Revista Brasileira de Psiquiatria. v. 26, n. Supl.1, p. 33-9, 2004.

KAPLAN, H. I.; SADOCK, B. J. Transtornos relacionados a substâncias. In: (Org) Compêndio de psiquiatria: ciências do comportamento e psiquiatria clínica. Porto Alegre: Artmed, 1997.

KAZDIN, A. E. Historia de la modificación de conduta. Bilbao: Desclée de Brouwer, 1983.

KNAPP, P. Terapia cognitivo-comportamental na prática psiquiátrica. Porto Alegre: Artmed, 2004.

KNAPP, P.; BECK, A. T. Fundamentos, modelos conceituais, aplicações e pesquisa da terapia cognitiva. Revista Brasileira de Psiquiatria, v. 30, n. Supl II, p. S54-64, 2008.

KOUIMTSIDIS, C. et al. Treatment or prison: service user and staff experiences of drug treatment and testing orders. Psychiatric Bulletin, v. 31, n. 12, p. 463-466, 2007.

LEAHY, R. L. Técnicas de terapia cognitiva: manual do terapeuta. Porto Alegre: Artmed, 2006.

LUSSIER, J. P. et al. A meta-analysis of voucher based reinforcement therapy for substance use disorders. Addiction, v. 101, n. 2, p. 192-203, 2006. 
MAHONEY, M. J. Cognition and behavior modification. Cambridge: Ballinger, 1974.

MARINHO, J. A. C. et al. Manejo da fissura. In: RIBEIRO, M.; LARANJEIRA, R. (Org.). O tratamento do usuário de crack. Porto Alegre: Artmed, 2012. p. 374-388.

MARLATT, G. A.; DONOVAN, D. M. (Org.). Prevenção de recaída: estratégias de manutenção no tratamento de comportamentos adictivos. Porto Alegre: Artmed, 2009.

MARLATT, G. A.; GORDON, G. R. Prevenção de recaída. Porto Alegre: Artmed, 1994.

MIGUEL, A. Q. C.; GAYA, C. M. Técnicas e terapias comportamentais aplicadas ao tratamento da dependência química. In: ZANELATTO, N. A.; LARANJEIRA, R. (Org.). O tratamento da dependência química e as terapias cognitivo-comportamentais: um guia para terapeutas. Porto Alegre: Artes Médicas, 2013. p. 313-329.

MILLER, W. R.; ROLLNICK, S. Entrevista Motivacional: Preparando as pessoas para a mudança de comportamentos adictivos. Porto Alegre: Artes Médicas, 2001.

MORAES, P. B.; BOSCHETTI, F. Técnicas cognitivas. In: ZANELATTO, N. A.; LARANJEIRA, R. (Org.). $O$ tratamento da dependência química e as terapias cognitivo-comportamentais: um guia para terapeutas. Porto Alegre: Artes Médicas, 2013. p. 293-310.

NORCROSS, J. et al. The face of 2010: a Delphi Poll on the future of psycotherapy. Professional Psychology: Research and Practice, v. 33, n. 3, p. 316, 2002.

RAMOS, S. P. Psicodinâmica. In: DIEHL, A. et al. Dependência química: prevenção, tratamento e políticas públicas. Porto Alegre: Artmed, 2011. p. 346-356.

RANGÉ, B. (Org.). Psicoterapias cognitivo-comportamentais: um diálogo com a psiquiatria. Porto Alegre: Grupo A, 2011.

RIBEIRO, M.; LARANJEIRA, R. (Org.). O tratamento do usuário de crack. Porto Alegre: Artmed, 2012.

ROBINS, R. W. et al. An empirical analysis of trends in psychology. American Psychologist, v. 54, n. 2, p. 117, 1999.

ROOZEN, H. G. et al. A systematic review of the effectiveness of the community reinforcement approach in alcohol, cocaine and opioid addiction. Drug and Alcohol Dependence, v. 74, n. 1, p. 113, 2004.

SCHOTTENFELD, R. S. et al. Community reinforcement approach for combined opioid and cocaine dependence: patterns of engagement in alternate activities. Journal of Substance Abuse Treatment, v. 18, n. 3, p. 255-261, 2000.

SEMERANI, A. Historia, teorias y técnicas de la psicoterapia cognitiva. Barcelona: Paidós, 2002.

SERRA, A. M. M. - Teoria e terapia cognitiva. In: ZANELATTO, N. A.; LARANJEIRA, R. (Org.). $O$ tratamento da dependência química e as terapias cognitivo-comportamentais: um guia para terapeutas. Porto Alegre: Artes Médicas, 2013. p. 106-121. 
SIEGEL, S. The role of conditioning in drug tolerance and addiction. In: KEEHN, J. D., editor. Psychopathology in animals: research and clinical implications. New York: Academic Press, 1979.

SILVA, C. J. A dependência química e o modelo cognitivo de Aaron Beck. In: ZANELATTO, N. A.; LARANJEIRA, R. (Org.). O tratamento da dependência química e as terapias cognitivocomportamentais: um guia para terapeutas. Porto Alegre: Artes Médicas, 2013. p. 122-134.

SIMON, R. Psicanálise e psicoterapia breve. Psicologia USP, v. 1, n. 1, p. 93-96, 1990.

SMITH, J. E. et al. The community reinforcement approach to the treatment of substance use disorders. The American Journal on Addictions, v. 10, n. s1, p. s51-s59, 2001.

VUCHINICH, R. E.; TUCKER, J. A. Contributions from behavioral theories of choice to na analysis of alcohol abuse. Journal of Abnormal Psychology, v. 97, n. 2, p. 181, 1988.

WORLD HEALTH ORGANIZATION. Classificação de transtornos mentais e de comportamento da CID-10: descrições clínicas e diretrizes diagnósticas. Porto Alegre: Artmed, 1993.

WRIGHT, J. H. et al. Aprendendo a terapia cognitivo-comportamental: um guia ilustrado. Porto Alegre: Artmed, 2008.

ZANELATTO, N. A.; LARANJEIRA, R. (Org.). O tratamento da dependência química e as terapias cognitivo-comportamentais: um guia para terapeutas. Porto Alegre: Artes Médicas, 2013.

Como citar este artigo (Formato ABNT):

MORANDI, M; GUIMARÃES, L.P. Intervenções Cognitivo-Comportamentais no Tratamento das Dependências Químicas. Id on Line Revista de Psicologia, Fevereiro de 2015, vol.9, n.25, p. 203-216. ISSN 1981-1189.

Recebido: 10/12/2014

Aceito:20/01/2015 